\title{
Drug Eluting Stents \& Coronary Angiography
}

\section{Dr. Bharat Rawat*, Dr. Pushpa Raj Poudel*, Dr. Smriti Mulmi*}

Twenty-five years ago, a young German physician, Andreas Gruentzig, inserted a catheter into a 38-year-old man's coronary artery, inflated a tiny balloon the doctor had fashioned in his own kitchen, successfully opening a blockage and restoring blood flow to a human heart. This event set a cascade of newer invention.

First performed in the mid-1980s, and approved by the U.S. Food and Drug Administration (FDA) in 1994, stenting is a catheter-based procedure in which a small, expandable wire mesh tube (stent) is inserted into a diseased artery as a scaffold to hold it open. Coronary artery stents have made it possible for physicians to treat diseased coronary arteries with less trauma to the patient than coronary artery bypass graft surgery. According to 2005 guidelines issued by the American Heart Association and American College of Cardiology, stents can be considered for use in patients who have significant disease of the left main and left anterior descending coronary arteries, the two largest coronary arteries. Patients with very serious coronary artery disease, or total blockage of major arteries, are still recommended for surgery.

Coronary artery stenting is almost always performed in conjunction with other catheterbased procedures, such as balloon angioplasty or atherectomy. These procedures are used to partially reduce the narrowing caused by atherosclerosis. In particular, stenting has been an important advance in balloon angioplasty. Before the introduction of stents, as many as half of all coronary arteries opened with a balloon-tipped catheter narrowed once again after the procedure (restenosis) in as much as $50 \%$ of cases. When they were first introduced, coronary stents were made from bare metal. These early generation stents greatly improved the success rate of balloon angioplasty, as measured by the restenosis rate. Restenosis occurs when the artery closes again after the procedure. The first bare-metal stents reduced the restenosis rate from about 50 percent to about 20 percent. By its nature, a stent is designed to be a permanent implant. In cases of complex or diffuse disease, multiple or even overlapping stents can be used, 


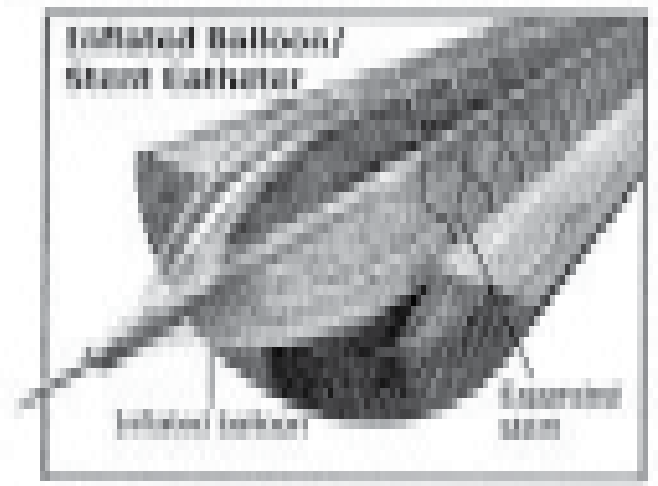

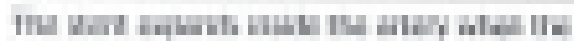

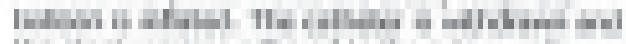

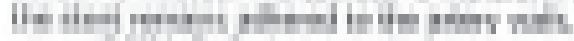

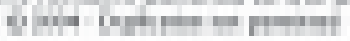

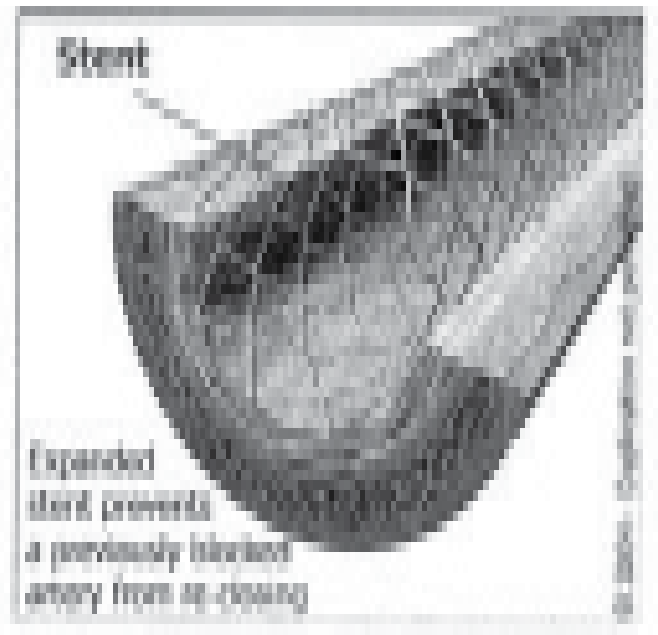

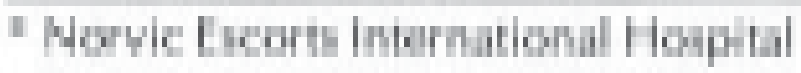

In 2003, however, a new generation of stents was introduced. These stents, called drugeluting stents, are covered with special drugs that reduced the restenosis rate to its current low rate $(<10 \%)$. The weight of evidence around DES to prove their superiority over bare metal stents clinically is overwhelming. Four year follow up data on DES (Cypher) shows a $67 \%$ reduction in target lesion revascularization rates compared to BMS. However the success of a stenting procedure can be compromised by risk factors such as smoking or high cholesterol levels, which could lead to new blockages in the coronary arteries, People receiving stents are strongly encouraged to learn and practice healthy lifestyle behaviors for good heart health.

In America Taxus and Cypher are two mostly used kind of DES which use anticancer drugs like Paclitaxel and Sirolimus respectively. These stents are believed to be good in diabetic patients. Medtronic which uses drug ABT-578 is widely used in Europe. Doctors are now viewing with heightened interest the problems of drug-eluting stent thrombosis, allergic inflammatory reactions, long term antiplatelet therapy and noncompliance. Over three years period, the cumulative rate of thrombosis of DES is $2.9 \%$, but what is disturbing is that the rate is linear starting at $1.2 \%$ at 30 days (similar to bare metal stents) and then $0.6 \%$ each year thereafter. Unlike bare-metal stents, thrombosis does not seem to wane with time, but continues to increase at the same rate. Another concern, perhaps related, has been that of hypersensitivity, or allergic reactions to drugeluting stents but sufficient data are unavailable regarding this concern. One of the

* Norvic Escorts International Hospital 
biggest concerns of cardiologists has been what type and duration of antiplatelet therapy is optimal after drug- eluting stents. After much study, aspirin, combined with ticlopidine (Tichid) and more recently clopidogrel (Plavix) became the primary antiplatelet drugs prescribed after stenting. Currently the FDA recommends 3 months for the Cypher stent, and 6 for the Taxus. However, because of their concerns, most cardiologists prescribe Plavix for a year and aspirin for life; some even prescribe Plavix for life.

The problems stated above are not unknown and not new, And a number of companies have been developing innovative technologies that avoid some of the problems with the first generation DES. For example, CoStar stent, which has small recesses in the stent struts that hold a bioabsorbable drug-eluting polymer. In two months, the drug is eluted and the polymer has disappeared and the stent has become, for all intents, a bare metal stent. Other companies, like Biotronik of Germany and Switzerland, are testing biodegradable stents that will be completely absorbed into the body.

Despite the current crop of devices, there have been rumblings in the US that hospitals may be returning to bare metal stents for certain purposes. It has been shown that there is no difference in the incidence of restenosis between a drug-eluting stent and a bare metal stent if the vessel was greater than $2.8 \mathrm{~mm}$. Thus it is logical that no patient who has a vessel greater than $2.8 \mathrm{~mm}$ should get a drug-eluting stent.

In view of the above mentioned development in cardiology our country Nepal is also catching its pace. Norvic Escorts International Hospital, in the heart of Kathmandu has always stood in the fore front in this regard. The first Cath Lab established in the year 2000 at Norvic has performed more than 200 angoplasties/stenting alone with a high success rate of $99 \%$. Now patients from Nepal don't need to go out of the country for treatment. The very high cost of surgery and logistics can now be saved. Also Norvic provides good patent care and personalized service at evidently cheap cost. Norvic promises to introduce state-of-the-art technologies in Nepal to lead interventional cardiac care to great heights.

The current level of cardiology interventions has been achieved only after a long period of consistent effort. The brief timeline below is intended to give an overview of the history of interventional cardiology. 
- 3000 B.C, - Egyptians perform bladder catheterizations using metal pipes.

- 400 B.C, - Catheters fashioned from hollow reeds and pipes are used in cadavers to study the function of cardiac valves.

- 1711 - Hales conducts the first cardiac catheterization of a horse using brass pipes, a glass tube and the trachea of a goose.

- 1844 - French physiologist Bernard coins the term "cardiac catheterization" and uses catheters to record intracardiac pressures in animals.

- 1929 - First documented human cardiac catheterization is performed by Dr. Werner Forssmann in Eberswald, Germany.

- 1956 - Forssmann, Cournand and Richards share the Nobel Prize, Cournand states in his acceptance speech "the cardiae catheter was...the key in the leek."

- 1958 - The diagnostic coronary angiogram - the key to selective imaging of the heart is discovered by Dr. Mason Sones

- 1964 - Transluminal Angioplasty, the concept of remodeling the artery, $1 \%$ introduced by Dr. Charles T. Dotter

- 1977 - Andreas Gruentzig performs first cath lab PTCA on awake patient in Zurich; starting with this case, all PTCA data is entered into a worldwide registry http://www. ptca. org/archive/bios/dotter.html

- 1982 - over-the-wire coaxial balloon systems introduced, brachial guiding catheters \& steerable guide wires are developed.

- 1994 - the Palmaz-Schatz stent is approved by the F.D.A. for use in the United States 
- 1997 - over one million angioplasties will be performed worldwide, making angioplasty the most common medical intervention in the world

- 2003 - The first drug-eluting stent, the Cypher, manufactured by Johnson \& Johnson / Cordis, is approved by the F.D.A., Marking a major advance in the battle to reduce restenosis to single digits

- 2004 - Boston Scientific gets its Taxus drug-eluting stent approved; many studies are published demonstrating the vastly improved outcomes from drug- eluting stents

\section{Total no of PTCA/Stents:222}

- Medicated Stents: 93,

- Non Medicated Stents:107,

- Single Stent: 177

- Double Stent: 18

- Three Stents: 1

- Four Stents: 1

To conclude, DES have revolutionized the way interventional cardiologists approach their patients and their treatment schedules. However bare metal stents may still be practical in some patients. Millions of heart patients all over the worldshall continue to take advantage by the newer technological advances in cardiology. 